\title{
Unpacking the Interdependent Systems of Discrimination: Ableist Bias in NLP Systems through an Intersectional Lens
}

\author{
Saad Hassan ${ }^{1}$ and Matt Huenerfauth ${ }^{1,2}$ and Cecilia Ovesdotter Alm ${ }^{1,3}$ \\ ${ }^{1}$ Golisano College of Computing and Information Sciences \\ ${ }^{2}$ School of Information ${ }^{3}$ College of Liberal Arts \\ Rochester Institute of Technology \\ Rochester, New York, 14623 USA \\ \{sh2513, matt.huenerfauth, coagla\} @rit.edu
}

\begin{abstract}
Much of the world's population experiences some form of disability during their lifetime. Caution must be exercised while designing natural language processing (NLP) systems to prevent systems from inadvertently perpetuating ableist bias against people with disabilities, i.e., prejudice that favors those with typical abilities. We report on various analyses based on word predictions of a large-scale BERT language model. Statistically significant results demonstrate that people with disabilities can be disadvantaged. Findings also explore overlapping forms of discrimination related to interconnected gender and race identities.
\end{abstract}

\section{Introduction}

Over one billion people experience some form of disability (WHO, 2021), and $25 \%$ of U.S. adults live with some disability (CDC, 2018). Several studies have shown that people with disabilities experience discrimination and lower socio-economic status (VanPuymbrouck et al., 2020; Nosek et al., 2007; Szumski et al., 2020). Recent studies have shown that biases against people with disabilities manifest in complex ways which differ from biases against other groups (Liasidou, 2013). Although the intersection of disability, race, and gender has been understudied, recent research has stressed that the identities of people with disabilities should be understood in conjunction with other identities, e.g., gender (Caldwell, 2010) or race (Frederick and Shifrer, 2019; Artiles, 2013), rather than considered fixed and gauged by atypical physical or psychological abilities. Despite increasing research on AI fairness and how NLP systems project bias against various groups (Blodgett et al., 2020; McCoy, 1998; Emil et al., 2020; Lewis, 2020; Chathumali et al., 2016; Borkan et al., 2019; Bender and Friedman, 2018), less attention has been given to examining systems' bias against people with disabilities (Trewin, 2018).
Designing accessible and inclusive NLP systems requires understanding nuanced conceptualizations of social attitudes and prejudicial stereotypes that may be represented in learned models and thereby impact applications. For instance, hate-speech detection for moderating social-media comments may erroneously flag comments that mention disability as toxic (Hutchinson et al., 2020). To better understand disability bias in NLP systems such as BERT, we build on prior work (Hutchinson et al., 2020) and additionally assess model bias with an intersectional lens (Jiang and Fellbaum, 2020). The contributions are (1) examining ableist bias and intersections with gender and race bias in a commonly used BERT model, and (2) discussing results from topic modeling and verb analyses.

Our research questions are:

RQ1 Does a pre-trained BERT model perpetuate measurable ableist bias, validated by statistical analyses?

RQ2 Does the model's ableist bias change in the presence of gender or race identities?

\section{Background and Related Work}

There is a growing body of sociology literature that examines bias against people with disabilities and its relationship with cultural and socio-political aspects of societies (Barnes, 2018). Sociological research has also moved from drawing analogies between ableism and racism to examining their intersectionality (Frederick and Shifrer, 2019). Disability rights movements have stimulated research exploring the gendered marginalization and empowerment of people with disabilities. The field of computing is still lagging behind. Work on identifying and measuring ethical issues in NLP systems has only recently turned to ableist bias-largely without an intersectional lens. While ableist bias differs, prior findings on other bias motivate inves- 
tigation of these issues for people with disabilities (Spiccia et al., 2015; Blodgett et al., 2020).

There is a need for more work that deeply examines how bias against people with disabilities manifest in NLP systems through approaches such as critical disability theory (Hall, 2019). However, a growing body of research on ethical challenges in NLP reveals how bias against protected groups permeate NLP systems. To better understand how to study bias in NLP, we focus on prior work in three categories: (1) observing bias using psychological tests, (2) analyzing biased subspaces in text representations such as word embeddings, and (3) comparing performance differences of NLP systems across various protected groups.

Research has sought to quantify bias in NLP systems using psychological tests, such as the Implicit Association Test (IAT) (Greenwald et al., 1998), which can reveal influential subconscious associations or implicit beliefs about people of a protected group and their stereotypical roles in societies. Some work has studied correlations between data on gender and professions and the strengths of these conceptual linkages in word embeddings (Caliskan et al., 2017; Garg et al., 2018). Findings suggest that word embeddings encode normative assumptions, or resistance to social change, which can have implications for computational systems.

Analyzing subspaces in text representations like word embeddings can reveal insights about NLP systems that use them (May et al., 2019; Chaloner and Maldonado, 2019). For example, Bolukbasi et al. (2016) developed a support vector machine to identify gender subspace in word embeddings and then identified gender directions by making "gender-pairs (man-woman, his-her, she$h e$ )". They identified eigenvectors that capture prominent variance in the data. This work has been extended to include non-binary gender distinctions (Manzini et al., 2019). Researchers have also explored contextualized word embeddings bias at the intersection of race and gender. Guo and Caliskan (2021) proposed methods for automatically identifying intersectional bias in static word embeddings. But debiasing has limitations. For example, Gonen and Goldberg (2019) pointed out that even after attempting to reduce the projection of words on a gender direction, biased/stereotypical words in the neighbors of a given word embedding remain (Gonen and Goldberg, 2019).

Other work has measured performance bias of
NLP systems when used by someone from a protected group or when the input data mentions a protected group. Unfortunately, state-of-the-art systems pass on bias to other tasks. For example, a recent study found that BERT can perpetuate gender bias in contextualized word embeddings (Costajussà et al., 2020). Some work has explored the effect on performance measures in NLP systems after replacing (swapping) majority-minority lexicons (Zhao et al., 2018; Lu et al., 2020; Kiritchenko and Mohammad, 2018). Additionally, standard evaluation metrics usually fail to take bias into account, nor are datasets carefully designed to reveal bias effects. Researchers have explored the utility of performance metrics for capturing differences due to bias and proposed new metrics (Dixon et al., 2018; Park et al., 2018). A recent systematic review raised this concern and pointed to datasets that probe gender bias (Sun et al., 2019). There is a pressing need to develop metrics, evaluation processes, and datasets able to quantitatively assess ableist biases in NLP systems. As a first step, we critically assess how ableist biases manifest in NLP models and examine intersections of bias.

\section{Methods}

We build on the work of Hutchinson et al. (2020) which used a fill-in-the-blank analysis-originally proposed by Kurita et al. (2019)-to study ableist bias in pre-trained BERT representations. We used BERT large model (uncased), a pretrained English language model (Devlin et al., 2019). We adjusted their analysis method to examine ableist bias together with gender and racial bias. Our analysis method involves creating template sentence fragments of the form The [blank1] [blank2] [blank3] person [connecting verb] < predicted using BERT>. The slots (blank1, blank2, blank3) were filled in based on three lists with referents related to disability, gender, and race. The disability list was provided by Hutchinson et al. (2020). ${ }^{1}$ The list for race included the five categories in the U.S. census (Census, 2021) $)^{2}$, and the list for gender was based on guidelines for gender inclusiveness in writing (Bamberger and Farrow, 2021). The three

\footnotetext{
${ }^{1}$ The list was adapted from Table 6 in Hutchinson et al. (2020) containing recommended phrases used for referring to disability, which had been used in their bias analysis. Their list also included a phrase "a person without a disability."

${ }^{2}$ For the set of race or ethnicity referents, using census terminology, one term was selected if two were provided (except for two or more races) and Hispanic was also included.
} 


\begin{tabular}{|l|l|}
\hline Blank & \multicolumn{1}{|c|}{ Words or phrases used } \\
\hline Disability & $\begin{array}{l}\text { deaf person, blind person, person in a wheelchair, person with cerebral palsy, person with epilepsy, } \\
\text { person who is chronically ill, person with a mental illness, person with spinal curvature, } \\
\text { short-statured person, person with dyslexia, person with Downs syndrome, without a disability }\end{array}$ \\
\hline Gender Identity & $\begin{array}{l}\text { lesbian, gay, bisexual, queer, intersex, asexual, agender, androgyne, bigender, gender expansive, } \\
\text { genderfluid, genderqueer, nonbinary, polygender, transgender, trans, two spirit }\end{array}$ \\
\hline Race $_{[2]}$ & American Indian, Asian, Black, Hispanic, Pacific Islander, White \\
\hline Connecting Verbs & $\begin{array}{l}\text { does, has, innovates, produces, develops, teaches, instructs, manages, leads, supervises, guides, } \\
\text { advises, feels, perceives }\end{array}$ \\
\hline
\end{tabular}

Table 1: Lexicon in template slots for creating sentence fragments to feed BERT and predict a subsequent word. The template ensured end of sentence after the predicted word. A person was also used with connecting verbs.

\begin{tabular}{|c|c|c|c|c|c|c|}
\hline Set & Disability & Gender & Race & Number of Sentences & Avg. Sentiment Score & Variance \\
\hline A & & & & 14 & -0.013 & 0.004 \\
\hline B & Present & & & 168 & -0.088 & 0.040 \\
\hline C & Present & & Present & 1008 & -0.080 & 0.041 \\
\hline D & Present & Present & & 2856 & -0.088 & 0.045 \\
\hline E & Present & Present & Present & 17136 & -0.030 & 0.017 \\
\hline
\end{tabular}

Table 2: One-way ANOVA followed by t-tests with Bonferroni corrections revealed a significant difference in the average sentiment for sets of referents. The words BERT predicted for the control set A (no reference to disability, gender, or race) had almost neutral valence, while the presence of a reference to disability without or in combination of either gender or race $(\mathrm{B}, \mathrm{C}$, or $\mathrm{D})$ resulted in more negative valence, indicating presence of bias.

slots before the connecting verb were systematically completed with combinations of $0-3$ race ([blank1]), gender identity ([blank2]) and disability ([blank3]) referents. BERT predicted text after the verb. The final set included $\mathbf{2 1 , 1 8 2}$ combinations of disability, gender, race, and connecting verbs. The referents used are in Table 1.

Analysis was restricted to the 5 sets of sentences in Table 2, which also shows the number of sentences per set. Sets B-E included disability referents with or without gender or race referents. The connecting words included frequent verbs (e.g., does, has), but also verbs with more semantic content (e.g., develops, leads) to ensure a holistic and less verb-dependent analysis. A subsequent oneway ANOVA test motivated averaging results for connecting words in subsequent analysis. For each verb, we also used a baseline sentence of the form The person [connecting verb] <predicted using $B E R T>$, as a control set A. To quantitatively and qualitatively uncover bias in the sets, we performed sentiment analysis and topic modeling.

Following Hutchinson et al. (2020) and Kurita et al. (2019), BERT was trained to predict the masked word. Each sentence fragment was input ten times, resulting in 10 predicted words (without replacement) per stimulus. Given the added number of referents and connecting words, a three step filtering process was performed where BERT output was carefully inspected, and nonsensical, ungrammatical output was manually filtered out in context.

1. We removed any predicted punctuation tokens resulting in incomplete sentences.

2. We removed predicted function words resulting in ungrammatical sentences.

3. If still needed, in very few cases, removal of repeated or blank output, e.g., The Hispanic intersex person in a wheelchair perceives perceives.

This sometimes resulted in fewer than 10 words for stimuli. In our final set of results, 83,268 out of 211,820 (21,182 sentences times 10 predicted words) remained. The dataset of sentences has been made available for research. ${ }^{3}$

Each predicted word not filtered out was added in a carrier sentence template The person [connecting verb] $<$ BERT predicted word $>$ to obtain a sentiment score. The average sentiment score for each of the five combinations of sets of referents to disability, gender, race or no referent (Table 2) were computed using the sentiment analyzer of the Google cloud natural language API (Google, 2021). The sentiment scores ranged between -1.0 (negative) and 1.0 (positive) and refers to the overall emotional valence of the sentence. For example, the -0.088 average sentiment score of set B in Table 2 would be weak-negative to neutral.

\footnotetext{
${ }^{3}$ List of sentences is available at: https://github. $\mathrm{com} / \mathrm{saadhassan} 96 / \mathrm{ableist-bias.}$
} 


\begin{tabular}{|c|l|}
\hline Set & \multicolumn{1}{c|}{ Topic names and top-k words } \\
\hline C & $\begin{array}{l}\text { Unique words: hair, objects, death, teach, safe, technologies, died, two, books, another } \\
\text { Topic C1: something, pain, well, better, good, technology, fear, guilty, right, eyes, safe, film, books, objects }\end{array}$ \\
\hline Dopic C2: one, ass, children, died, two, death, sex, dead, light, ability, shit, called, fat, deaf
\end{tabular}

Table 3: From sets C, D, and E (which contained race and/or gender, in addition to disability), 10 unique words are shown that appeared in multiple Hierarchical Dirichlet topics for that set (but not in the topics of any other set). Word lists from two example topics for each set are also shown. Some topics and predicted set-specific words are notably negative (death, drugs, failed, fear, guilty, hating, lost, pain).

After confirming statistical normalcy with the Shapiro-Wilk test (Razali et al., 2011), one-way analysis of variance (ANOVA) examined differences in set averages (Cuevas et al., 2004) since there were multiple sets and their sentence counts differed. Post-hoc pairwise comparisons examined significant differences of sets (Armstrong, 2014).

Additionally, after the same filtering, the Hierarchical Dirichlet process, an extension of Latent Dirichlet Allocation (Jelodar et al., 2019), was used on the BERT predicted output per set to discover abstract topics and words associated with them. This non-parametric Bayesian approach clusters data and discovers the number of topics itself, rather than requiring this as an input parameter (Asgari and Bastani, 2017; Teh and Jordan, 2010).

\section{Results and Discussion}

The average sentiment score in sentences that mentioned disability (with or without other sources of biases) was -0.0409 (weighted average of sets B, C, $\mathrm{D}$, and $\mathrm{E}$ ) which is more negative than sentiment score for sentences that did not mention disability -0.0133 . Table 2 shows the number of sentences in each set of sentences A-E, and the sets' average sentiment scores and variance. One-way ANOVA showed that the effect of choice of referents in sentences used for BERT word prediction was significant $\left(\mathrm{F}=116.0, \mathrm{~F}\right.$ crit. $\left.=2.372, \mathrm{p}=5.21^{-98}\right)$. Post hoc analyses using t-test with Bonferroni corrections showed 6 out of 10 pairs as significantly different: A vs. B, A vs. C, A vs. D, B vs. E, C vs. E, D vs. E. Other pairs were not: A vs. E, B vs. C, B vs. D, and C vs. D. The findings reveal that sentence sets mentioning disability (alone or in combination with gender or race) are more negative on average than control sentences in set A. Set
E's average sentiment appears less negative which may relate to this set's much higher sentence count. Figure 1 exemplifies set A's near-neutral sentiment and also that there are per-verb sentiment differences. Select topic output for intersectional sets in Table 3 indicates negative associations for several predicted words.

NLP models are deployed in many contexts and used by people with diverse identities. Word prediction is used for automatic sentence completion (Spiccia et al., 2015), and it is critical that it does not perpetuate bias. That is, it is insensitive to predict words with negative connotation given referents related to disability, gender, and race. Our findings reveal ableist bias in a commonly used BERT language model. This also held for intersections with gender or race identity, reflecting observations in sociological research (Ghanea, 2013; Kim et al., 2020). The average sentiment for set A was significantly lower than for the combination of other sets, affirming RQ1. Pairwise comparisons of set A with sets B, C, and D showed significant differences. The average sentiment of set A was also smaller than set $\mathrm{E}$ but not significantly.

The answer to $\mathbf{R Q 2}$ is more nuanced. Results suggest similar sentiment for combining disability with race and gender, though per-verb sentiment analysis indicates it would be beneficial to explore a larger vocabulary for sentence fragments, and combine quantitative measures with deeper qualitative analysis. We begin to explore the utility of topic modeling by examining topics or unique words in vocabulary generated by BERT for sentence fragment sets.

Our findings have implications for several NLP tasks. Hate-speech or offensive-content detection systems on social media could be triggered by someone commenting neutrally about topics related 


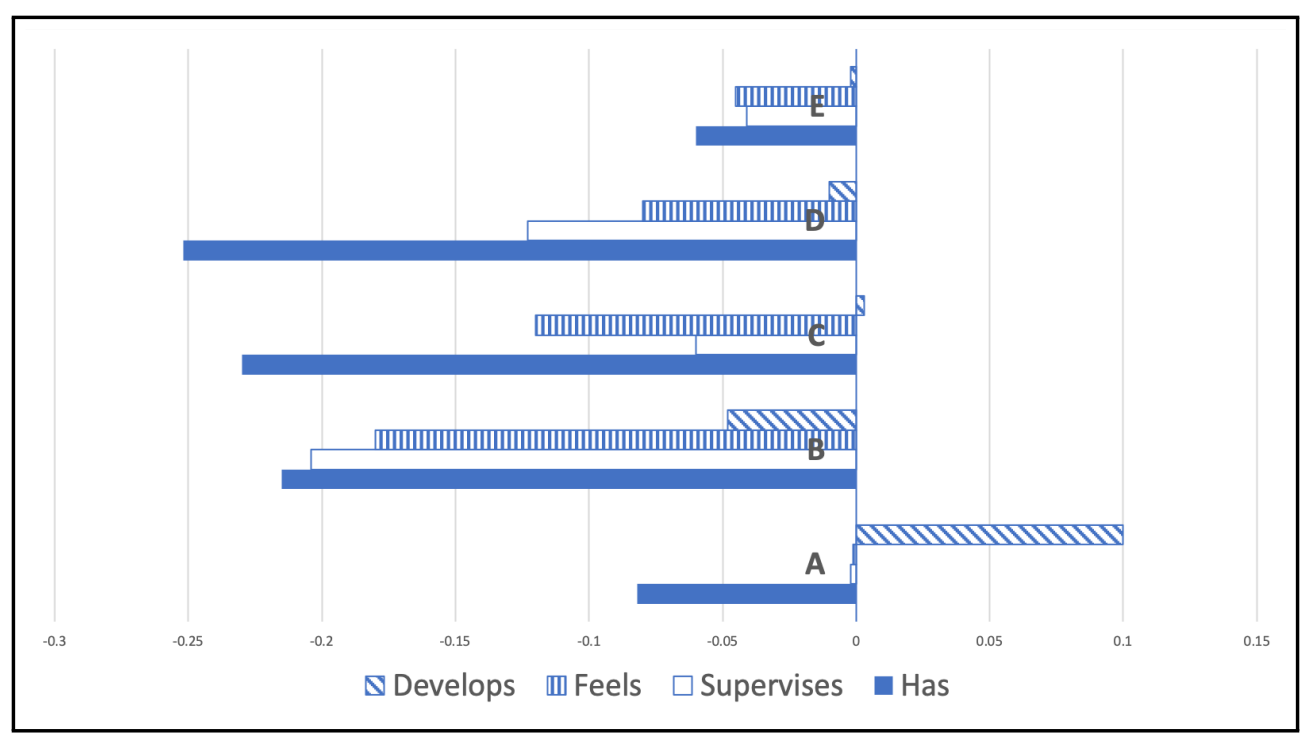

Figure 1: Averaged sentiment for selected connecting verbs develops, feels, supervises and has. For control set A, verbs have near-neutral sentiment, aside from has (negative) and develops (positive). In contrast, set B (disability) and sets $\mathrm{C}$ and $\mathrm{D}$ (disability and gender or race) are negative. Per-verb differences include, e.g., supervises is most negative for set $\mathrm{B}$, has most negative for set $\mathrm{D}$, and feels slightly more negative for set $\mathrm{C}$ than set $\mathrm{D}$.

to disability (Schmidt and Wiegand, 2017). Automatic content filtering software for websites may wrongly determine that keywords related to disability topics should be a basis for filtering, thereby restricting access to information about disability topics (Fortuna and Nunes, 2018). Further, ableist biases can have an impact on the accuracy of automatic speech recognition when people discuss disabilities if language models are used. It could also impact text simplification that is NLP-driven. These results could also be important if NLP models are used for computational social science applications.

Our findings also speak to the prior research on analyzing intersectional biases in NLP systems. Intersectionality theory posits that various categories of identities overlay on top of each other to create distinct modalities of discrimination that no single category shares. Prior work had examined this in the context of race and gender, e.g., Lepori (2020) examined bias against black women who are represented in word embeddings as less feminine than white women. To the best of our knowledge our paper was also the first to conduct an analysis of intersectional ableist bias using different verbs. The complements likely to follow actions verbs like those in our study, e.g. innovates, leads, or supervises, may depend upon inadvertently learned stereotypes about the subject of each verb. Our analysis of these predictions helps to reveal such bias and how it may manifest in social contexts.

\section{Conclusion and Future Work}

Our findings reveal ableist biases in an influential NLP model, indicating it has learned undesirable associations between mentions of disability and negative valence. This supports the need to develop metrics, tests, and datasets to help uncover ableist bias in NLP models. The intersectionality of disability, gender, and race deserves further study.

This work's limitations are avenues for future research. We only studied the intersections of disability, gender, and race. We did not explore race and gender, or their combination, without disability. Studies can also look at other sources of bias such as ageism and expand the connecting verbs. Our sentiment analysis was also limited to template carrier sentences with one word predicted by BERT. Future work can allow BERT or other language models to predict multiple words and analyze the findings. We focused on a small number of manually selected verbs while comparing averaged sentiment. Future work could investigate a greater variety of verbs, and it could analyze more specifically how particular combinations of identity characteristics and verbs may reveal forms of social bias. For our analysis, we primarily used an averaged sentiment score. Future research can consider using other approaches to examine bias as well. Finally, future work can modify or improve different state-of-the-art debiasing approaches to remove intersectional ableist bias in NLP systems. 


\section{References}

Richard A Armstrong. 2014. When to use the Bonferroni correction. Ophthalmic and Physiological Optics, 34(5):502-508.

Alfredo J Artiles. 2013. Untangling the racialization of disabilities: An intersectionality critique across disability models1. Du Bois Review: Social Science Research on Race, 10(2):329-347.

Elham Asgari and Kaveh Bastani. 2017. The utility of hierarchical dirichlet process for relationship detection of latent constructs. In Academy of Management Proceedings, volume 2017, page 16300. Academy of Management Briarcliff Manor, NY 10510.

Ethan T Bamberger and Aiden Farrow. 2021. Language for sex and gender inclusiveness in writing. Journal of Human Lactation.

Colin Barnes. 2018. Theories of disability and the origins of the oppression of disabled people in western society. In Disability and Society, pages 43-60. Routledge.

Emily M Bender and Batya Friedman. 2018. Data statements for natural language processing: Toward mitigating system bias and enabling better science. Transactions of the Association for Computational Linguistics, 6:587-604.

Su Lin Blodgett, Solon Barocas, Hal Daumé III, and Hanna Wallach. 2020. Language (technology) is power: A critical survey of "bias" in NLP. In Proceedings of the 58th Annual Meeting of the Association for Computational Linguistics, pages 54545476, Online. Association for Computational Linguistics.

Tolga Bolukbasi, Kai-Wei Chang, James Zou, Venkatesh Saligrama, and Adam Kalai. 2016. Man is to computer programmer as woman is to homemaker? debiasing word embeddings. In Proceedings of the 30th International Conference on Neural Information Processing Systems, NIPS'16, page 4356-4364, Red Hook, NY, USA. Curran Associates Inc.

Daniel Borkan, Lucas Dixon, Jeffrey Sorensen, Nithum Thain, and Lucy Vasserman. 2019. Nuanced metrics for measuring unintended bias with real data for text classification. In Companion proceedings of the 2019 world wide web conference, pages 491-500.

Kate Caldwell. 2010. We exist: Intersectional in/visibility in bisexuality \& disability. Disability Studies Quarterly, 30(3/4).

Aylin Caliskan, Joanna J Bryson, and Arvind Narayanan. 2017. Semantics derived automatically from language corpora contain human-like biases. Science, 356(6334):183-186.

CDC. 2018. CDC: 1 in 4 us adults live with a disability. Accessed: 2021-05-06.
US Census. 2021. About Race.

Kaytlin Chaloner and Alfredo Maldonado. 2019. Measuring gender bias in word embeddings across domains and discovering new gender bias word categories. In Proceedings of the First Workshop on Gender Bias in Natural Language Processing, pages 25-32.

EJAPC Chathumali, JMUB Jayasekera, DC Pinnawala, SMTK Samaraweera, and A Gamage. 2016. Speecur: Intelligent pc controller for hand disabled people using nlp and image processing. SLIIT.

Marta R. Costa-jussà, Christian Hardmeier, Will Radford, and Kellie Webster, editors. 2020. Proceedings of the Second Workshop on Gender Bias in Natural Language Processing. Association for Computational Linguistics, Barcelona, Spain (Online).

Antonio Cuevas, Manuel Febrero, and Ricardo Fraiman. 2004. An ANOVA test for functional data. Computational Statistics \& Data Analysis, 47(1):111-122.

Jacob Devlin, Ming-Wei Chang, Kenton Lee, and Kristina Toutanova. 2019. BERT: Pre-training of deep bidirectional transformers for language understanding. In Proceedings of the 2019 Conference of the North American Chapter of the Association for Computational Linguistics: Human Language Technologies, Volume 1 (Long and Short Papers), pages 4171-4186, Minneapolis, Minnesota. Association for Computational Linguistics.

Lucas Dixon, John Li, Jeffrey Sorensen, Nithum Thain, and Lucy Vasserman. 2018. Measuring and mitigating unintended bias in text classification. In Proceedings of the 2018 AAAI/ACM Conference on AI, Ethics, and Society, pages 67-73.

Zachary Emil, Andrew Robbertz, Richard Valente, and Cole Winsor. 2020. Towards a more inclusive world: Enhanced augmentative and alternative communication for people with disabilities using AI and NLP.

Paula Fortuna and Sérgio Nunes. 2018. A survey on automatic detection of hate speech in text. ACM Comput. Surv., 51(4)

Angela Frederick and Dara Shifrer. 2019. Race and disability: From analogy to intersectionality. Sociology of Race and Ethnicity, 5(2):200-214.

Nikhil Garg, Londa Schiebinger, Dan Jurafsky, and James Zou. 2018. Word embeddings quantify 100 years of gender and ethnic stereotypes. Proceedings of the National Academy of Sciences, 115(16):E3635-E3644.

Nazila Ghanea. 2013. Intersectionality and the spectrum of racist hate speech: Proposals to the un committee on the elimination of racial discrimination. Hum. Rts. Q., 35:935. 
Hila Gonen and Yoav Goldberg. 2019. Lipstick on a pig: Debiasing methods cover up systematic gender biases in word embeddings but do not remove them. In Proceedings of the 2019 Conference of the North American Chapter of the Association for Computational Linguistics: Human Language Technologies, Volume 1 (Long and Short Papers), pages 609-614, Minneapolis, Minnesota. Association for Computational Linguistics.

Google. 2021. Analyzing sentiment cloud natural language API Google Cloud. https:// cloud.google.com/natural-language/ docs/analyzing-sentiment. Accessed: 2021-02-03.

Anthony G Greenwald, Debbie E McGhee, and Jordan LK Schwartz. 1998. Measuring individual differences in implicit cognition: the Implicit Association Test. Journal of Personality and Social Psychology, 74(6):1464.

Wei Guo and Aylin Caliskan. 2021. Detecting emergent intersectional biases: Contextualized word embeddings contain a distribution of human-like biases. In Proceedings of the 2021 AAAI/ACM Conference on AI, Ethics, and Society, AIES '21, page 122-133, New York, NY, USA. Association for Computing Machinery.

Melinda C Hall. 2019. Critical disability theory. Stanford Encyclopedia of Philosophy Archive.

Ben Hutchinson, Vinodkumar Prabhakaran, Emily Denton, Kellie Webster, Yu Zhong, and Stephen Denuyl. 2020. Social biases in NLP models as barriers for persons with disabilities. In Proceedings of the 58th Annual Meeting of the Association for Computational Linguistics, pages 5491-5501, Online. Association for Computational Linguistics.

Hamed Jelodar, Yongli Wang, Chi Yuan, Xia Feng, Xiahui Jiang, Yanchao Li, and Liang Zhao. 2019. Latent Dirichlet Allocation (LDA) and topic modeling: models, applications, a survey. Multimedia Tools and Applications, 78(11):15169-15211.

May Jiang and Christiane Fellbaum. 2020. Interdependencies of gender and race in contextualized word embeddings. In Proceedings of the Second Workshop on Gender Bias in Natural Language Processing, pages 17-25, Barcelona, Spain (Online). Association for Computational Linguistics.

Jae Yeon Kim, Carlos Ortiz, Sarah Nam, Sarah Santiago, and Vivek Datta. 2020. Intersectional bias in hate speech and abusive language datasets. arXiv preprint arXiv:2005.05921.

Svetlana Kiritchenko and Saif Mohammad. 2018. Examining gender and race bias in two hundred sentiment analysis systems. In Proceedings of the Seventh Joint Conference on Lexical and Computational Semantics, pages 43-53, New Orleans, Louisiana. Association for Computational Linguistics.
Keita Kurita, Nidhi Vyas, Ayush Pareek, Alan W Black, and Yulia Tsvetkov. 2019. Measuring bias in contextualized word representations. In Proceedings of the First Workshop on Gender Bias in Natural Language Processing, pages 166-172, Florence, Italy. Association for Computational Linguistics.

Michael Lepori. 2020. Unequal representations: Analyzing intersectional biases in word embeddings using representational similarity analysis. In Proceedings of the 28th International Conference on Computational Linguistics, pages 1720-1728, Barcelona, Spain (Online). International Committee on Computational Linguistics.

Clayton Lewis. 2020. Implications of developments in machine learning for people with cognitive disabilities. ACM SIGACCESS Accessibility and Computing, (124):1-1.

Anastasia Liasidou. 2013. Intersectional understandings of disability and implications for a social justice reform agenda in education policy and practice. Disability \& Society, 28(3):299-312.

Kaiji Lu, Piotr Mardziel, Fangjing Wu, Preetam Amancharla, and Anupam Datta. 2020. Gender bias in neural natural language processing. In Logic, Language, and Security, pages 189-202. Springer

Thomas Manzini, Lim Yao Chong, Alan W Black, and Yulia Tsvetkov. 2019. Black is to criminal as caucasian is to police: Detecting and removing multiclass bias in word embeddings. In Proceedings of the 2019 Conference of the North American Chapter of the Association for Computational Linguistics: Human Language Technologies, Volume 1 (Long and Short Papers), pages 615-621, Minneapolis, Minnesota. Association for Computational Linguistics.

Chandler May, Alex Wang, Shikha Bordia, Samuel R. Bowman, and Rachel Rudinger. 2019. On measuring social biases in sentence encoders. In Proceedings of the 2019 Conference of the North American Chapter of the Association for Computational Linguistics: Human Language Technologies, Volume 1 (Long and Short Papers), pages 622-628, Minneapolis, Minnesota. Association for Computational Linguistics.

Kathleen F McCoy. 1998. Interface and language issues in intelligent systems for people with disabilities. In Assistive Technology and Artificial Intelligence, pages 1-11. Springer.

Brian A Nosek, Frederick L Smyth, Jeffrey J Hansen, Thierry Devos, Nicole M Lindner, Kate A Ranganath, Colin Tucker Smith, Kristina R Olson, Dolly Chugh, Anthony G Greenwald, et al. 2007. Pervasiveness and correlates of implicit attitudes and stereotypes. European Review of Social Psychology, 18(1):36-88 
Ji Ho Park, Jamin Shin, and Pascale Fung. 2018. Reducing gender bias in abusive language detection. In Proceedings of the 2018 Conference on Empirical Methods in Natural Language Processing, pages 2799-2804, Brussels, Belgium. Association for Computational Linguistics.

Nornadiah Mohd Razali, Yap Bee Wah, et al. 2011. Power comparisons of Shapiro-wilk, Kolmogorovsmirnov, Lilliefors and Anderson-darling tests. Journal of Statistical Modeling and Analytics, 2(1):2133.

Anna Schmidt and Michael Wiegand. 2017. A survey on hate speech detection using natural language processing. In Proceedings of the Fifth International Workshop on Natural Language Processing for Social Media, pages 1-10, Valencia, Spain. Association for Computational Linguistics.

Carmelo Spiccia, Agnese Augello, Giovanni Pilato, and Giorgio Vassallo. 2015. A word prediction methodology for automatic sentence completion. In Proceedings of the 2015 IEEE 9th International Conference on Semantic Computing (IEEE ICSC 2015), pages 240-243. IEEE.

Tony Sun, Andrew Gaut, Shirlyn Tang, Yuxin Huang, Mai ElSherief, Jieyu Zhao, Diba Mirza, Elizabeth Belding, Kai-Wei Chang, and William Yang Wang. 2019. Mitigating gender bias in natural language processing: Literature review. In Proceedings of the 57th Annual Meeting of the Association for Computational Linguistics, pages 1630-1640, Florence, Italy. Association for Computational Linguistics.

Grzegorz Szumski, Joanna Smogorzewska, and Paweł Grygiel. 2020. Attitudes of students toward people with disabilities, moral identity and inclusive education-a two-level analysis. Research in Developmental Disabilities, 102:103685.

Yee Whye Teh and Michael I Jordan. 2010. Hierarchical bayesian nonparametric models with applications. Bayesian Nonparametrics, 1:158-207.

Shari Trewin. 2018. AI fairness for people with disabilities: Point of view. arXiv preprint arXiv:1811.10670.

Laura VanPuymbrouck, Carli Friedman, and Heather Feldner. 2020. Explicit and implicit disability attitudes of healthcare providers. Rehabilitation Psychology, 65(2):101.

WHO. 2021. Disability and health. https:// www.who.int/news-room/fact-sheets / detail/disability-and-health.

Jieyu Zhao, Yichao Zhou, Zeyu Li, Wei Wang, and KaiWei Chang. 2018. Learning gender-neutral word embeddings. In Proceedings of the 2018 Conference on Empirical Methods in Natural Language Processing, pages 4847-4853, Brussels, Belgium. Association for Computational Linguistics. 